\title{
DETECCIÓN DE RESIDUOS DE AFLATOXINAS EN HÍGADO DE POLLO EN MERCADOS DE LIMA
}

\author{
Detection of aflatoxin residues in chicken liver in lima markets \\ Arbaiza F. Teresa ${ }^{1}$, Córdova R. Augusta ${ }^{2}$, Icochea D. Eliana ${ }^{3}$, Cutti Hilda ${ }^{4}$ \\ ${ }^{1}$ D.A. Producción Animal. Facultad de Medicina Veterinaria. UNMSM. ${ }^{2}$ D.A. Farmacología, Bromatología y Nutrición. \\ Facultad de Farmacia y Bioquímica. UNMSM. ${ }^{3}$ D.A. Salud Animal y Salud Pública. Facultad de Medicina Veterinaria. \\ UNMSM. ${ }^{4}$ Bachiller Medicina Veterinaria. UNMSM.
}

\section{RESUMEN}

Las aflatoxinas causan una variedad de alteraciones en las aves, influyendo en la patología hepática (aflatoxicosis). Siendo el hígado el más afectado, al ser expendidos en mercados de alimentos, ponen en riesgo la salud del consumidor por los probables residuos de aflatoxinas. El objetivo de este estudio fue detectar la presencia de aflatoxinas en hígado de pollos procedentes de los mercados de abastos de Lima. Se muestrearon 16 mercados de diferentes sectores socio económicos de Lima. De cada mercado se subclasificaron hígados normales y aparentemente sospechosos. El análisis de las 32 muestras se realizó en el Laboratorio de Bioquímica, Nutrición y Alimentación Animal de la Facultad de Medicina Veterinaria de la UNMSM. Se empleó para la detección de aflatoxinas el método de ELISA, que cuenta con la aprobación del Research Institute of AOAC, empleando el "kit" Veratox HS ${ }^{\circledast}$ (alta sensibilidad o, 1, 2, 4 y 8 ppb). Por ser los hígados muestras muy complejas, se utilizó para la extracción columnas de inmunoafinidad "Neo Column ${ }^{\circledast ”}$ para aflatoxinas (o4/o8). El límite de detección fue de o,5 ppb. No se detectó aflatoxinas en los hígados analizados. Se asume que el aspecto de los hígados subclasificados como sospechosos, podría tener origen en otros procesos metabólicos y que la presencia de secuestrantes en los alimentos de pollos tiene buen rendimiento.

Palabras clave: aflatoxinas, aflatoxicosis, hígado de pollo.

\section{SUMMARY}

Aflatoxins cause a variety of disorders in poultry, especially affecting the liver (aflatoxicosis). Contaminated livers may become a health risk to consumers when expended in food markets. The purpose of the present study was to detect the presence of aflatoxins in chicken livers in Lima food markets. Samples were collected in 16 markets located in various socioeconomic sectors of Lima. From every market livers were subclassified as normal and apparently suspicious. Analysis of 32 samples was conducted at the Laboratory of Biochemistry, Nutrition and Animal Feeding, Veterinary Medicine Faculty, San Marcos University. Aflatoxins were determined through the ELISA method approved by the AOAC Research Institute, using the Veratox HS (high sensitivity, o, 1, 2, 4, and 8 ppb). Liver samples are quite complex so immunoaffinity extraction columns (NEOCOLUMN for aflatoxins, 04/o8) were used. The detection limit was $0.5 \mathrm{ppb}$. None of the sampled was detected with aflatoxins. The aspect of livers considered as suspicious might be due to other metabolic processes than aflatoxin contamination, but also to the good performance of micotoxin adsorbents in food.

Key words: Aflatoxins, Aflatoxicosis, Chiken liver.

\section{INTRODUCCIÓN}

$\mathrm{E}$ 1 conocimiento científico de los alimentos y los controles de calidad son elementos fundamentales para la salud comunitaria; dentro de este contexto debe considerarse la evaluación de los riesgos toxicológicos y los grados de incidencia originados por micotoxinas. Los hongos productores de aflatoxinas se encuentran en una variedad de alimentos, especialmente vegetales, de gran importancia en la dieta humana y animal, como son cereales y oleaginosas. Cuando no existe un control adecuado en la alimentación animal, se presentan cuadros agudos de aflatoxicosis originados por diversidad de micotoxinas en desmedro de la producción, en términos económicos y de reducción de fuentes alimentarias para la población. Para el ser humano, el mayor riesgo es la toxicidad crónica asociada al consumo de pequeñas cantidades de toxinas durante período prolongado ${ }^{(1)}$.

Los residuos de micotoxinas en el organismo animal no sólo implican que el animal ingiera un alimento contaminado con los consiguientes riesgos de toxicidad, sino también el peligro para los humanos que los consuman. Básicamente, existen dos vías de intoxicación: la primaria, cuando se consume directamente 
un alimento contaminado y la secundaria cuando se ingieren residuos de micotoxinas presentes en la carne, vísceras, huevos o leche ${ }^{(2)}$.

La aflatoxina es un contaminante común de los alimentos, especialmente en las dietas básicas de muchos países en vías de desarrollo. Esta toxina es producida por la acción micótica durante las etapas de producción, cosecha, almacenamiento y procesamiento del alimento, y es considerada un contaminante inevitable de los alimentos por la Food and Drug Administration (FDA) de USA ${ }^{(3)}$.

La aflatoxina $\mathrm{B}_{1}$ es absorbida vía tracto gastrointestinal dentro del sistema portal sanguíneo y llevada al hígado donde se metaboliza. Una porción es activada y fijada en los tejidos hepáticos. Algunos metabolitos conjugados de la aflatoxina $B_{1}$, que son solubles en agua, se excretan por la bilis y consecuentemente van a las heces. Otros conjugados (también solubles en agua) y productos de degradación de aflatoxina $B_{1}$, al igual que los metabolitos no conjugados de ésta, son transferidos al sistema circulatorio y distribuidos sistémicamente. Eventualmente, los mencionados residuos van a la leche, huevos, músculo y tejidos comestibles ${ }^{(4)}$.

El daño de la síntesis proteica y la disminución de la facilidad del organismo para movilizar las grasas están relacionados aparentemente con la lesión hepática (necrosis y cambios grasos) que presentan los animales afectados por aflatoxinas en forma precoz. En el interior de los hepatocitos, las aflatoxinas se unen a macromoléculas tales como DNA, puntos endoplasmáticos para fijación de esteroides y diversas enzimas. El primer cambio producido por aflatoxina $\mathrm{B}_{1}$ observado en ratas es la modificación de la estructura del nucleolo del hepatocito; la lesión de éste es compatible con la unión observada entre las aflatoxinas y el DNA nuclear. Entre los cambios estructurales posteriores se incluyen la disgregación y reducción en el número de ribosomas, la proliferación del retículo endoplasmático liso, la pérdida de glucógeno y la degeneración de las mitocondrias. El efecto inmunológico de las aflatoxinas parece ser una depresión humoral no específica y que, en parte, produce una alteración en los anticuerpos tisulares ${ }^{(5)}$.

En Japón, Bintvihok y col. (2002), realizaron estudios sobre la presencia de residuos de aflatoxina en hígado, músculo y huevos de aves domésticas, para lo cual alimentaron durante siete días a patos, gallinas ponedoras, codornices y pollos "broiler" con dietas que contenían 3 ppm de aflatoxina $\mathrm{B}_{1}\left(\mathrm{AFB}_{1}\right)$. Las aves fueron sacrificadas entre el octavo y decimoprimer día de alimentación con $\mathrm{AFB}_{1}$. Las aflatoxinas y sus metabolitos fueron determinados por HPLC. Los niveles de $\mathrm{AFB}_{1}$ en los tejidos de codornices fueron más altos que en las otras aves. En todas las especies estudiadas, las aflatoxinas y sus metabolitos fueron 10 veces más altas en el hígado que en el músculo ${ }^{(6)}$.

En nuestro país, el consumo de vísceras de ave tiene gran aceptación en diferentes presentaciones culinarias; por lo que el objetivo de este trabajo fue detectar residuos de aflatoxinas en hígado de pollos que se expenden en Lima, para lo cual se obtuvo muestras procedentes de mercados formales de los diferentes distritos.

\section{PARTE EXPERIMENTAL}

Plan de muestreo.- Las muestras de hígado de pollo se adquirieron en 16 mercados de abastos de Lima, distribuidos entre los diferentes sectores socioeconómicos, en los distritos: Miraflores, La Molina, San Borja, Magdalena, Jesús María, Lince, Breña, Surquillo, San Martín de Porras, Lima Metropolitana (Mercado Central), Rimac, Cercado de Lima (La Aurora), La Victoria, San Juan de Miraflores, Villa El Salvador y Villa María del Triunfo.

Preparación de la muestra.- En el Laboratorio de Bioquímica, Nutrición y Alimentación Animal se procedió al diagnóstico para discriminar hígados normales y aparentemente sospechosos de cada mercado, obteniéndose un total de 32 muestras para la detección de aflatoxinas. El tamaño de la muestra fue de 500 gramos, divididos en porciones de $50 \mathrm{~g}$, de hígados normales y $50 \mathrm{~g}$. de aparentemente sospechosos.

\section{Detección y cuantificación de aflatoxinas totales}

Extracción de la toxina.- Los 50 g de cada una de las muestras se maceraron con 6 g de cloruro de sodio, luego se licuaron con $100 \mathrm{~mL}$ de metanol al $70 \%$ con Phosphate Buffered Salina (PBS) y $50 \mathrm{~mL}$ de n-hexano durante tres minutos. La masa heterogénea se filtró por papel de filtración rápida, se dejó en reposo, y la segunda filtración se realizó por papel Wathman № 4. Los extractos se pasaron por las columnas de inmunoafinidad $(04 / 08)^{(7)}$. Se utilizó el método de ELISA, que cuenta con la aprobación de Research Institute of AOAC (1995) empleando el "kit" cuantitativo Veratox ${ }^{\circledR}$ - Alta Sensiblidad de la Corporación NEOGEN USA ${ }^{(8)}$. El ensayo emplea un ELISA de tipo competitivo directo cuya sensibilidad es de o, $5 \mathrm{ppb}$, realizándose todas las pruebas por duplicado. 


\section{MATERIALES Y MÉTODOS}

48 micropozos de mezclados marcados con una línea de color rojo.

40 micropozos cubiertos con anticuerpo monoclonal.

Controles de aflatoxinas de 1 a 8 ppb

Conjugado: aflatoxina - HRP

Sustrato TMB: Tetrametil benzidina

Solución de detección de la reacción

\section{Procedimiento:}

- Se transfirió $100 \mu \mathrm{l}$ del conjugado a los pozos de mezclados

- Se transfirió $100 \mu \mathrm{l}$ del control y de la muestra problema en cada pozo.

- Se transfirió $100 \mu \mathrm{l}$ del conjugado a los pozos sin marca. Se incubará por 10 minutos.

- Se descartó el líquido de los pozos con anticuerpos.

- Se lavó 5 veces con agua desionizada.

- Se golpeó los pozos contra papel absorbente.

- Se adicionó $100 \mu \mathrm{l}$ de sustrato a los pozos. Se incubará por 10 minutos.

- Se añadió $100 \mu \mathrm{l}$ de la solución de detección de la reacción a todos los pozos.

- El color desarrollado se cuantificó en el lector Biotek EL-301 para pozos, espectrofotómetro que mide la densidad óptica (OD650) de cada pozo utilizado en la prueba.

- Los resultados analíticos se procesaron en el programa Neogen para computadora. Se determinó los niveles de aflatoxinas en cada muestra en términos de absorbancia transfiriéndose a ppb $(\mu \mathrm{g} / \mathrm{kg})$.

\section{RESULTADOS Y DISCUSIÓN}

Realizadas las lecturas de las absorbancias de los controles y muestras problemas se obtuvo los resultados analíticos que se detallan en la Tabla 1.

No teniendo resultados positivos en las muestras problemas, se asume que el aspecto de los hígados sospechosos tendría origen en otra alteración metabólica.

El síndrome de hígado graso está considerado como una enfermedad metabólica y esporádica de aves obesas enjauladas y explotadas en climas calurosos; se registra en aves de alta producción, alimentadas con raciones elevadas de energía, que presentan exceso de tejido adiposo en la cavidad abdominal, hígado y riñón, aumentan su volumen, se tornan pálidos, friables y de color amarillo graso ${ }^{(9)}$.
Tabla 1. Concentración de aflatoxinas HS en ppb $\left({ }^{*}\right)$

\begin{tabular}{ccc}
\hline Estandar ppb & Densidad óptica & ppb \\
\hline 0 & 0,741 & 0,0 \\
1 & 0,609 & 1,0 \\
2 & 0,517 & 2,1 \\
4 & 0,413 & 4,2 \\
8 & 0,316 & 7,6 \\
\hline
\end{tabular}

*Aflatoxinas de alta sensibilidad en partes por billón (ppb).

El tratamiento de los alimentos de la industria avícola, generalmente con secuestrantes de aflatoxinas, tendría un buen rendimiento.

Los especialistas en avicultura tienen la certeza del impacto negativo de las micotoxinas en la productividad; debido a esto, el uso de los adsorbentes de micotoxinas se ha generalizado en la industria avícola. Muchos tipos de aluminosilicatos comerciales, cuando son incluidos en la dieta, ofrecen gran protección contra las aflatoxinas, más no contra las demás micotoxinas; en este caso la alternativa más viable es el retiro del alimento contaminado.

Diversos trabajos de investigación concluyen que el uso de adsorbentes es una ayuda efectiva a la solución de problemas de productividad y alimentos contaminados ${ }^{(10)}$.

\section{CONCLUSIÓN}

En las 32 muestras de hígado de pollos analizadas procedentes de 16 mercados de abastos de Lima no se detectó presencia de aflatoxinas.

\section{REFERENCIAS BIBLIOGRÁFICAS}

1. Arbaiza T. Riesgos de la contaminación con aflatoxina en maní (Arachis hipogea) de producción nacional. Tesis de Magister en Bromatología. Facultad de Farmacia y Bioquímica, Universidad Nacional Mayor de San Marcos. Lima - Perú. 1998. 62 p.

2. Basílico JC. 1995. Micotoxinas en alimentos. Centro de Publicaciones Universidad Nacional Del Litoral. Santa Fe - Argentina. Pag: 8-9.

3. FAO. DIGESA, 2004. Taller Nacional Sobre Criterios del CODEX para el Establecimiento de límites máximos permitidos para aditivos, contaminantes, residuos de plaguicidas y medicamentos de uso veterinario en alimentos. Lima Perú. 3-6. Febrero del 2004. Revisado el 25 de Marzo 2009. Disponible en htpp://www.rlc.fao.org/ prior/comagric/codex/rla2904/pdf/lmpper.pdf.

4. GimenoAand Martins ML. Mycotoxinsand Mycotoxicosis in Animals and Human - Special Nutrition Inc. USA. Ed. México 2006. pp 1-127. 
5. Perusia OR y Rodríguez AR. Micotoxicosis. Rev. Inv. Perú 2001; 12(2): 87-116.

6. Bintvihok A, Theingnin S, Doiks, Residues of Aflatoxins in the liver, muscle anu eggs of domes the fowls. J. Vet Med 2002. 64 (11): 1037 - 39.

7. Neogen Europe Ltd 2009. Neo Column For Aflatoxin (Wide bore) o4/o8.

8. Neogen Corporation 2008 Veratox(x) HS Quantitative Aflatoxin High Sensivity Test, (USA/Canadá).

9. Baez AJ Patología de las Aves. ira Edición. México. Editorial Trillas 1994. 138 p.

10. Kubena LF, Harvey RB, et al. Hill. Efficacy of Hydrated Sodium Calcium aluminiosilicate to reduce the toxicity of Aflatoxin and Diacetoxysciroenol. Poultry Sci: 1993. 72: 51-59.

\section{Agradecimiento}

Al M.V. Juan Carlos Flores - LAPROVET EIRL, a la Dra. Amanda Chávez de García - Facultad de Medicina Veterinaria UNMSM, a la Srta. Lourdes Lagos P. y al Sr. Armando Arbaiza F. por su apoyo en la ejecución del proyecto.

Manuscrito recibido el: 30/11/2009

Aceptado para su publicación el:07/01/2010

\section{Correspondencia:}

Dra. Arbaiza Fernández Teresa Sebastiana

Dirección: Av. Javier Mariategui 538 - Jesús María

Correo electrónico: tarbaiza@yahoo.es 